\title{
Simulation of Deformable Objects Using Sliding Mode Control with Application to Cloth Animation
}

\author{
Farshad Rum and Brandon W. Gordon \\ Control and Information Systems (CIS) Laboratory, Department of Mechanical and \\ Industrial Engineering, Concordia University, Montreal, Quebec, Canada, \\ bwgordon@me. concordia.ca
}

\begin{abstract}
A new method is presented for simulation of deformable objects that consist of rigid and flexible structural elements using the control based Singularly Perturbed Sliding Manifold (SPSM) approach. The problem is multi-scale due to its rigid and flexible components and forms a set of differential-algebraic equations. The problem is formulated as a set of ODEs with inequality constraints by allowing some deviations in the rigid links. The SPSM approach is particularly well suited for such problems. It is shown that this method can handle inconsistent initial conditions, and it allows the user to systematically approximate the equations due to its robustness properties. The inherent attractivity of the sliding dynamics enables the method to handle sudden changes in position, velocity or acceleration while satisfying geometrical constraints. The desired level of accuracy in constraint errors is achieved in a finite time and thereafter. Moreover, the new approach is explicit and capable of performing multi-rate and real-time simulations. Finally, it is shown that the SPSM approach to simulation requires the inversion of a smaller matrix than comparable implicit methods. The result is significantly improved performance for computationally expensive applications such as cloth animation.
\end{abstract}

\section{Introduction}

Animation of deformable structures such as hair, chain, cloth and jelly-type materials has imposed some challenging problems due to their multi-scale nature. Such problems have little resistance in bending/shear directions, but are often very stiff with hard constraints in elongation/shear directions. The resulting set of equations is therefore stiff and traditional explicit methods usually cannot handle them efficiently due to small time steps that they demand.

Implicit methods on the other hand can handle larger time steps; however, they have no built-in mechanism to deal with algebraic constraints. For a visually realistic animation of cloth a maximum deviation of $10 \%$ in the stiff direction (usually elongation) is recommended [1]; otherwise, the cloth will become like rubber. Traditionally it has been up to the user to select proper parameters in the stiff direction so that deviations do not exceed their limit. Such an approach might require a lot of trial and errors and if a large gain is necessary it can substantially limit the allowable time step, which was the reason for incorporating the method in the first place. 
Another approach has been to first solve the equations by imposing and correcting the constraints [2]. Such a method requires additional overhead and can result in artifacts that may in turn require another algorithm to correct the momentum/energy alterations caused by enforcing the constraints [3]. The proposed SPSM approach, however, has a built-in mechanism [4] to handle the limits on the constraints while at the same time solving the set of ODEs. Another merit of this approach is that due to its attractive boundary layer [5] it can be combined with existing codes that require sudden corrections in position, velocity or acceleration of particles in order to satisfy various geometrical constraints.

As we show in Sect. 2 the governing equations of a flexible object can be easily written as a set of ordinary differential equations in bending and shear direction and constrained by a set of algebraic equations in elongation directions. This class of equations is commonly referred to as differential-algebraic (DAEs) equations. In general, one can write such equations as:

$$
\dot{\mathbf{x}}=\mathbf{f}(t, \mathbf{x}, \mathbf{z}), \mathbf{0}=\mathbf{g}(t, \mathbf{x}, \mathbf{z})
$$

with $\mathbf{x} \in \mathfrak{R}^{n}, \mathbf{z} \in \mathfrak{R}^{m}, \mathbf{f}: \mathfrak{R} \times \mathfrak{R}^{n} \times \mathfrak{R}^{m} \rightarrow \mathfrak{R}^{n}$, and $\mathbf{g}: \mathfrak{R} \times \mathfrak{R}^{n} \times \mathfrak{R}^{m} \rightarrow \mathfrak{R}^{m}$. Where nonlinear functions $\mathbf{f}$ and $\mathbf{g}$ respectively represent the ODE part and algebraic constraints of the DAE.

In most cases the accuracy level required for constraints allows us to permit a certain amount of error, $\mathcal{E}$. Therefore the above set of DAEs can be relaxed to the following set of ODEs with algebraic inequalities:

$$
\dot{\mathbf{x}}=\mathbf{f}(t, \mathbf{x}, \mathbf{z}),\left|\mathbf{g}_{i}(t, \mathbf{x}, \mathbf{z})\right| \leq \varepsilon_{i}, i=1, \ldots, m
$$

where $\mathbf{g}_{i}(t, \mathbf{x}, \mathbf{z})$ represents each algebraic constraint and $m$ is the total number of them. The common amount of $\varepsilon$ used in simulation of cloth objects is $10 \%$ of the rest length in that link.

Singularly perturbed sliding manifold, SPSM, is a recent method developed to particularly attack problems of the type given by equations (2). SPSM equations can be efficiently solved by any explicit method and this allows us to perform a multi-rate simulation [6]; moreover, this approach is object-oriented thus any simulation code developed by this approach can be easily integrated with other simulation codes [6] SPSM realization is a robust method that allows us to ignore various terms and make efficient simulations in a systematic manner. Reaching phase and locking properties of the sliding control [5] translate into two desirable properties in our application. Firstly the fact that inconsistent initial conditions that are an issue for BDF methods [7] are systematically dealt with; in fact it is possible to show that after a finite time the bounds on errors are satisfied [5] and secondly the locking property of the sliding control assures us that once these bounds are reached they will be satisfied for the rest of the simulation.

Finally as we will see in Sect. 3 SPSM method has to invert a smaller size matrix at each time step compared to BDF methods. 


\section{Problem Formulation}

We model the flexible object as a collection of distributed masses connected to each other via rigid/flexible connections, which is also referred to as a particle system. These models have the ability to capture complex dynamical behaviors and are well suited to animation needs [3]. All the forces either internal or external simply depend on location and velocity of particles therefore in order to simulate such systems we only need to compute forces on each particle and two simple integrations will yield positions and velocities.

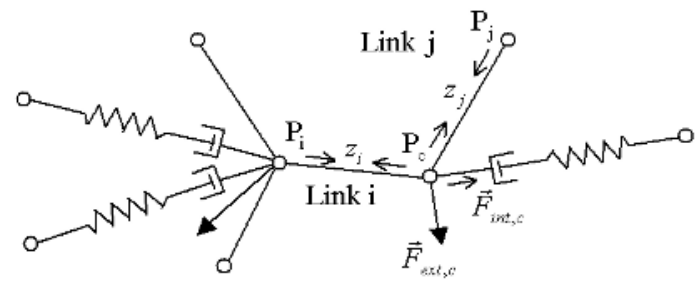

Fig. 1. A generic 3D section of a flexible object modelled as a particle system

As shown in Fig. 1 we can generally categorize the forces on particles as internal forces due to flexible connections, $\overrightarrow{\mathbf{F}}_{\text {int }}$, that are responsible for shear and bending behavior of the object, internal forces due to rigid connections, $z$, and finally the external forces, $\overrightarrow{\mathbf{F}}_{\text {ext }}$, that represent interaction forces between the object and the environment such as collisions, contacts, gravity, wind, etc. Take note that we are not modeling the rigid links as springs but rather keep their forces as unknowns for the controller to determine. As a result we have the following ODE:

$$
\left\{\begin{array}{l}
\dot{\overrightarrow{\mathbf{R}}}_{k}=\overrightarrow{\mathbf{V}}_{k} \\
\overrightarrow{\dot{\mathbf{V}}}_{k}=\frac{1}{m_{k}}\left(\sum_{j} z_{j} \hat{\mathbf{r}}_{k j}+\overrightarrow{\mathbf{F}}_{i n t, k}+\overrightarrow{\mathbf{F}}_{e x t, k}\right), k=1, \ldots, n_{p}
\end{array}\right.
$$

with these constraints

$$
0=L_{i}-L_{i}^{0}, \quad i=1, \ldots, n_{l}
$$

Here $\overrightarrow{\mathbf{R}}_{k}$ and $\overrightarrow{\mathbf{V}}_{k}$ represent the position and velocity of each particle, $n_{p}$ is the number of particles, $\hat{\mathbf{r}}_{k j}$ is the unit vector from particle $k$ to $j$ that is at the other end of the rigid link connecting them. For constraints, $L_{i}$ is the instantaneous length of the $i^{\text {th }}$ link, $L_{i}^{0}$ is its desired length and $n_{l}$ represents the total number of links.

By permitting the length of links to change as much as $\varepsilon_{i}$ the constraints will be the following inequalities:

$$
\left|L_{i}-L_{i}^{0}\right| \leq \varepsilon_{i}, \quad i=1, \ldots, n_{l} .
$$


We start application of the SPSM method by introducing the following error variable:

$$
w_{i}=L_{i}-L_{i}^{0}
$$

Differentiating w.r.t. time (see Fig. 1) one obtains:

$$
\dot{w}_{i}=\hat{\mathbf{r}}_{c i} \cdot\left(\overrightarrow{\mathbf{V}}_{i}-\overrightarrow{\mathbf{V}}_{c}\right), \ddot{w}_{i}=\frac{\left\|\overrightarrow{\mathbf{V}}_{i}-\overrightarrow{\mathbf{V}}_{c}\right\|^{2}-\dot{w}_{i}^{2}}{L_{i}}+\hat{\mathbf{r}}_{c i} \cdot\left(\overrightarrow{\dot{\mathbf{V}}}_{i}-\overrightarrow{\dot{\mathbf{V}}}_{c}\right) \text {. }
$$

Since

$$
\overrightarrow{\dot{\mathbf{V}}}_{c}=\frac{1}{m_{c}}\left(\sum_{j} z_{j} \hat{\mathbf{r}}_{c j}+\overrightarrow{\mathbf{F}}_{i n t, c}+\overrightarrow{\mathbf{F}}_{e x t, c}\right)
$$

We can see that $z$ terms appear in $\ddot{w}$. Therefore, according to definition of index of a DAE [7] our problem is of index three. The sliding surface designed by the SPSM method will then be:

$$
s_{i}=\mu^{2} \ddot{w}_{i}+2 \mu \dot{w}_{i}+w_{i}
$$

where $\mu$ is a positive parameter that determines the dynamics of the fast motion. The SPSM method then designs a controller that forces the motion to the above desired dynamics. In order to see the effect of $\mu$ on error we recall the following result from $[5]$ :

Lemma 1. If $\left|s_{i}(t)\right| \leq \varepsilon_{i}$ for $t \geq t_{r}$ then the error and its derivatives are bounded by:

$$
\left|w_{i}(t)\right| \leq \varepsilon_{i},\left|\dot{w}_{i}(t)\right| \leq \frac{2 \varepsilon_{i}}{\mu} \text { and }\left|\ddot{w}_{i}(t)\right| \leq \frac{4 \varepsilon_{i}}{\mu^{2}} \text { for } t \geq t_{r} .
$$

Note that in the above lemma we did not assume that initial conditions are necessarily consistent. Sliding mode control guarantees that after a finite reaching time, $t_{r}$, the motion will be contained within the desired accuracy bound, $\left|s_{i}(t)\right| \leq \varepsilon_{i}$, and the locking property guarantees that the motion will satisfy the required bounds ever after [5]. This in fact not only solves our ODE problem with inequality constraints, $\left|w_{i}(t)\right| \leq \varepsilon_{i}$, but also keeps the error derivatives bounded after a finite reaching phase with no need for the initial conditions to be consistent.

In order to achieve the above goals we design a controller that determines the value of $\mathbf{v}=\dot{\mathbf{z}}$. The value of the link forces, $\mathbf{z}$, will then be obtained by integrating $\mathbf{v}$.

Differentiating $\ddot{w}_{i}$ w.r.t time and packing the $\dddot{\mathbf{w}}$ vector we can write:

$$
\dddot{\mathbf{w}}=\mathbf{J}_{\Omega} \mathbf{v}+\boldsymbol{\beta}, \mathbf{J}_{\Omega}=\frac{\partial \ddot{\mathbf{w}}}{\partial \mathbf{z}}
$$

Substituting in eq. (9) we obtain: 


$$
\dot{\mathbf{s}}=\mu^{2} \ddot{\mathbf{w}}+2 \mu \ddot{\mathbf{w}}+\mathbf{w}=\mu^{2} \mathbf{J}_{\Omega} \mathbf{v}+\mu^{2} \boldsymbol{\beta}+2 \mu \ddot{\mathbf{w}}+\mathbf{w}=\mathbf{J}_{s} \mathbf{v}+\boldsymbol{\alpha}
$$

where

$$
\mathbf{J}_{s}=\mu^{2} \mathbf{J}_{\Omega}, \boldsymbol{\alpha}=\mu^{2} \boldsymbol{\beta}+2 \mu \ddot{\mathbf{w}}+\mathbf{w} .
$$

If we solve the above equation for $\mathbf{v}$ we can steer the sliding motion into the desired boundary layer. At this stage we incorporate ideas from sliding modes control and make use of its robustness properties. Since computation of exact $\boldsymbol{\alpha}$ can potentially be expensive we approximate it by $\hat{\boldsymbol{\alpha}}$. Moreover, it is possible to avoid inverting the original $\mathbf{J}_{S}$ that is potentially very large when we have a large number of rigid links in the object and use an approximate inverse denoted by $\hat{\mathbf{J}}_{S}^{-1}$. In [4] Gordon shows that if we compute $\mathbf{v}$ by the following controller:

$$
\mathbf{v}=-\hat{\mathbf{J}}_{s}^{-1}\left(\hat{\boldsymbol{\alpha}}+\mathbf{K} \operatorname{diag}\left[\operatorname{sat}\left(\frac{s_{i}}{\varepsilon_{i}}\right)\right]\right)
$$

the motion will converge to its desired error bound after a short reaching phase and will stay there ever after, if the following conditions are satisfied:

$$
\text { 1- } \mathbf{J}_{s} \hat{\mathbf{J}}_{s}^{-1} \mathbf{K}-\left|\operatorname{diag}\left[\boldsymbol{\alpha}-\mathbf{J}_{s} \hat{\mathbf{J}}_{s}^{-1} \hat{\boldsymbol{\alpha}}\right]\right| \text { has to be positive diagonally dominant. }
$$

2- $\mathbf{J}_{s} \hat{\mathbf{J}}_{s}^{-1} \mathbf{K}$ must be uniformly positive definite.

The sat(.) function used in eq. (14) is indeed the linear saturation function and is used to smooth the control and help us avoid the chattering phenomenon [5] common to sliding mode control methods.

In this work we simply invert the real jacobian matrix, $\hat{\mathbf{J}}_{s}^{-1}=\mathbf{J}_{s}^{-1}$, thus reducing (15) to:

$1-\mathbf{K}-|\operatorname{diag}[\boldsymbol{\alpha}-\hat{\boldsymbol{\alpha}}]|$ has to be positive diagonally dominant.

2- $\mathbf{K}$ must be uniformly positive definite.

The above criteria can then be easily satisfied by a large enough $\mathbf{K}$. All we are left with is choosing $\hat{\boldsymbol{\alpha}}$ and computing the $\mathbf{J}_{s}$ matrix.

The exact expression for $\boldsymbol{\alpha}$ is given by eq. (13). One can notice that $\ddot{\mathbf{w}}$ and $\mathbf{w}$ have already been evaluated in the process of calculating $\mathbf{s}$ and choice of

$$
\hat{\boldsymbol{\alpha}}=2 \mu \ddot{\mathbf{w}}+\mathbf{w}
$$

does not involve much computational overhead. If we further take our gain matrix to be diagonal, $\mathbf{K}=\operatorname{diag}\left(k_{i}\right)$, the only sufficient condition we need to meet becomes:

$$
k_{i} \geq \mu^{2}\left|\beta_{i}\right|
$$


Given the fact that $\beta_{i}$ mainly depends on the ODE (3) that is under control we do not have to retune the gains each time we try a new value for $\mu$.

Consider the generic link $i$ in Fig. 1 and the particle, $\mathrm{P}_{\mathrm{c}}$ connecting it to link $j$. Using definition (11) with equations (7) and (8) yields:

$$
\left[\mathbf{J}_{\Omega}\right]_{i, j}= \begin{cases}-\hat{\mathbf{r}}_{c i} \cdot \hat{\mathbf{r}}_{c j} / m_{c} & \text { if } i \neq j \\ -1 / m_{i 1}-1 / m_{i 2} & \text { if } i=j \text { and none of the two end particles } \\ & \text { of link } i \text { has an acceleration constraint } \\ -1 / m_{i, \text { free }} & \text { if } i=j \text { and only one end particle of link } i \\ 0 & \text { does not have an acceleration constraint } \\ \text { if links } i \text { and } j \text { have no nodes in common }\end{cases}
$$

In the above equation masses of the two end particles of link $i$ are denoted by $m_{i 1}$ and $m_{i 2}$ and mass of the particle in link $i$ that does not have an acceleration constraint is represented by $m_{i \text {,free }}$. Examples of an acceleration constraint could include when the particle is fixed at its place or when it is attached to another object, which is considerably more massive compared to the cloth. In the latter situation the acceleration of the attaching particle is mainly governed and constrained by the corresponding point in that object, for example consider the attachment points in animation of parachute or sail for cloth, or the connecting point of hair to an object.

Finally take note that the size of the jacobian matrix that has to be inverted is equal to the number of rigid links, $n_{l}$. We will make use of this fact in Sect. 3 .

Remark 1. In simulations that we performed, the algorithm proved to be robust against programming errors that yielded a slightly wrong $\mathbf{J}_{s}$. Aside from the fact that an amount of error in permitted by eq. (15), if the user makes a mistake in recognizing if a particle's acceleration is or is not constrained, the constraint could be considered as a neglected external force on that particle. This simply induces an error in $\hat{\boldsymbol{\alpha}}$ and as demonstrated by (16) can be robustly cancelled by choosing a big $\mathbf{K}$, which apparently does not involve any additional overhead. This fact can be especially handy when our flexible object dynamically changes its connections with other objects, e.g. when the sails are torn and taken away by a strong wind! A more common case happens in interactive animations where some points of the flexible object are dynamically chosen and moved by the user. We successfully tested this idea in our simulations presented in Sect. 3 .

\section{Application to Cloth Animation}

We adopt the structure proposed by Provot [2] that has proven effective in cloth animation [3]. The particles are rigidly linked to their adjacent horizontal and vertical particles. Shear spring-dampers attach immediate diagonally adjacent particles and finally bending characteristics are modeled by inserting spring and dampers between every other horizontally or vertically neighboring nodes. 
In animations we study a $n_{r}$ by $n_{c}$ grid of particles and we set the maximum amount of deviation in rigid links to be less than $10 \%$ of their intended lengths. As mentioned in Sect. 2 in all simulations we simplified the programming task by ignoring constraints in particles in evaluation of $\mathbf{J}_{s}$.

In order to test the new algorithm we initially compressed the left side of the cloth and fixed it at its two left corners. This is a typical example of inconsistent initial conditions that commonly arise in cloth animation, e.g. to attach pieces of cloth to rigid bodies. It is obvious that finding a consistent set of initial conditions that locates particles in proper positions to give this simple geometric shape is potentially tedious. In our example we exerted a tiny impulse to one of the particles and the algorithm automatically located all particles in proper positions that satisfied all constraints (Figs. 2).



(a)

(b)

(c)

Fig. 2. Testing the reaching phase of the algorithm by inconsistent initial conditions (a) before and (b) after a finite reaching time and subject to wind and gravity (c) subject to an external geometrical constraint

In simulations the motion reaches the desired boundary layer in at most 2 seconds for the link with the most initial deviation. We also tested the algorithm simultaneously under gravity, wind and an external object that imposed geometrical perturbations as shown in Figs. 2.

In order to compare the SPSM approach with the well-established BDF method we run a number of simulations using the popular implicit method proposed in [8]. The results of simulations are presented in table (1). For simplicity we used a constant step size in all simulations. The set of equations in both cases are sparse and the CG method [9] is used in all simulations. Note that eq. (19) shows that $\mathbf{J}_{s}$ is symmetric and sparse with a maximum of seven non-zero elements on each row; because each link is at most connected to six other ones.

As shown in table (1) the SPSM method always has a smaller matrix size. Given a $n_{r}$ by $n_{c}$ grid of particles the number of links equals $n_{l}=n_{r}\left(n_{c}-1\right)+n_{c}\left(n_{r}-1\right)$ and the number of particles is $n_{p}=n_{r} n_{c}$. The size of the matrix in implicit method [8] is three times the number of particles, $3 n_{p}=3 n_{c} n_{r}$, compared to the size of the $\mathbf{J}_{s}$, which is equal to the number of links or $n_{l}=2 n_{c} n_{r}-n_{c}-n_{r}$. Table 1 summarizes the results of simulations on an $8 \times 8$ grid of particles for 20 seconds. Simulations were run on a $2519 \mathrm{MHz}$ processor. Note that in order to avoid instability in the implicit 
method we had to reduce the amount of initial perturbations (left side compression) to one-third the amount shown in Figs. 2. It is evident from these results that the proposed approach is much more computationally efficient than standard implicit methods.

Table 1. Simulation results for SPSM and the implicit method [8]

\begin{tabular}{|c|c|c|c|}
\hline Method & $\begin{array}{c}\text { CPU } \\
\text { time }(\mathrm{sec})\end{array}$ & $\begin{array}{c}\text { Size of the sparse } \\
\text { system }\end{array}$ & $\begin{array}{c}\text { Max. stable cte. step } \\
\text { size }(\mathrm{sec})\end{array}$ \\
\hline Implicit & 41.12 & 192 & 0.002 \\
\hline SPSM & 5.47 & 112 & 0.019 \\
\hline
\end{tabular}

\section{Conclusions}

In this paper we have studied the problem of simulating a deformable object consisting of rigid and flexible inner connections using sliding mode control. As our case study we developed a code that simulated a piece of cloth under initial and continuous disturbances and compared it to a popular implicit method. The new approach was seven times faster, more than three times more robust to disturbances, handled almost ten times larger time steps and finally led to a sparse system with nearly half the size of the implicit method.

\section{References}

1. House, D., Breen ,D. E., (eds.): Cloth Modeling and Animation. A.K. Peters, Natick Mass. (2000)

2. Provot, X.: Deformation Constraints in a Mass-Spring Model to Describe Rigid Cloth Behaviour. Proc. Graphics Interface 95 (1995) 147-154

3. Desbrun, M., Meyer, M., Barr, A.H.: Interactive Animation of Cloth-Like Objects for Virtual Reality. Journal of Vizualisation and Computer Animation (2000)

4. Gordon, B.W.: State Space Modelling of Differential-Algebraic Systems using Singularly Perturbed Sliding Manifolds. Ph.D Thesis, MIT, Mechanical Engineering Dept., August (1999)

5. Slotine, J.-J.E.: Sliding Controller Design for Nonlinear Systems. Int. J. Control. 40 (1984) 2

6. Gu, B., Asada, H.H.: Co-Simulation of Algebraically Coupled Dynamic Subsystems. ACC (2001) 2273-2278

7. Brenan, K., Campbell, S., Petzold, L.: Numerical Solution of Initial Value Problems in Differential-Algebraic Equations. Amsterdam, North-Holland (1989)

8. Baraff, D., Witkin, A..: Large Steps in Cloth Simulation. In: Cohen, M. (ed.): SIGGRAPH 98 Conference Proceedings. Annual Conference Series, P. Addison-Wesley, July (1998) 43-54

9. Shewchuk, J.: An Introduction to the Conjugate Gradient Method Without the Agonizing Pain. Technical report CMU-CS-TR-94-125, Carnegie Mellon University (1994) 